\title{
Effect of heat treatment parameters on the microstructure and microhardness of Ti- 6Al-4V alloy
}

\begin{abstract}
Ti-6Al-4V is a dual-phase ( $\left.\breve{U}_{+} 6\right)$ Ti-alloy which possesses potential series and complex microstructures. The coexistence of 6 -phase alongside Ŭ-phase in Ti-6Al-4V alloy enhances the heat treatment process. Precise adjustments of heat treatment parameters can lead to diversity of microstructures that can be transformed from equiaxed to fully lamellar to bimodal. These microstructures have a critical impact on the mechanical properties. This work investigates the effect of altering the heat treatment parameters on both the microstructure and microhardness of Ti-6Al-4V alloy to elucidate alloyôs behaviour on the basis of microstructure - properties relations. Recrystallization annealing, solution treatment followed by aging, and 6 -annealing were performed on several samples to obtain various microstructures. The as-received sample exhibited fine equiaxed structure with a grain size of $1.78 \mu \mathrm{m}$. Recrystallization annealing of the fine equiaxed structure yielded considerable grain growth, resulting $7.29 \mu \mathrm{m}$ larger globular grains. The bi-modal microstructure was obtained from the equiaxed structure through solution treatment followed by aging. The application of b-annealing treatment resulted in a lamellar microstructure. The microhardness readings were affected by variations in the heat treatment procedures. The highest and lowest hardness were $386.1 \mathrm{Hv}$ and $302.2 \mathrm{Hv}$ for the lamellar and the equiaxed microstructures, respectively. The improvement in the microhardness was $27.8 \%$. In comparison, the bi-modal microstructure demonstrated a balanced hardness.
\end{abstract}

Keyword: Heat treatment; Microhardness; Microstructure; Ti-6Al-4V alloy 